\title{
SEMI-GROUPS AND DIFFERENTIAL EQUATIONS
}

\author{
J. D. GRAY
}

(Received 5 February 1968; revised 21 May 1968)

In this short note we shall apply the theory of semi-groups of operators, (cf: Hille and Phillips, [2]), to the problem of representing solutions of certain differential equations with non-constant coefficients. When the coefficients are constant, this representation reduces to the usual Laplace transform solution of the relevant equation.

Let $C[0, \infty]$ be the Banach space of continuous, real-valued functions defined on the closed interval $[0, \infty]$, with the usual pointwise algebraic operations, and with the supremum norm. Suppose that $a \in C[0, \infty]$ is a positive function. Define the function $A$ by

$$
A(t)=\int^{t} \frac{d s}{a(s)} .
$$

Then certainly $A$ is strictly increasing, so $A^{-1}$ exists. Further, as $A^{\prime}(t)=1 / a(t)>0$ for $t \geqq 0$, we see that $A^{-1}$ is in fact continuous. Now, for each $\xi \geqq 0$, define the function $q_{\xi}$ by

$$
q_{\xi}(t)=A^{-1}(A(t)+\xi) ; t \geqq 0 .
$$

Then $\left\{q_{\xi}: \xi \geqq 0\right\}$ is a family of continuous fractional iterates of $q_{1}$, and $A$ is the Abel function of this family, ([3]). Furthermore, because of (1), the limit

$$
\lim _{\xi \rightarrow 0+} \frac{\partial}{\partial \xi} q_{\xi}(t)
$$

exists for each $t \geqq 0$, and equals $a(t)$. Therefore $a$ is the infinitesimal generator of the iterates $\left\{q_{\xi}\right\}$.

Next, consider the mapping $W(\xi): C[0, \infty] \rightarrow C[0, \infty]$ defined, for each non-negative $\xi$, by $(W(\xi) x)(t)=x\left(q_{\xi}(t)\right)$, for $x \in C[0, \infty]$ and $t \geqq 0$. Obviously $W(\xi)$ is a linear mapping and it is readily seen that $W(\xi) W(\eta)=W(\xi+\eta)$ for all $\xi, \eta \geqq 0$. So the family $\mathfrak{W}=\{W(\xi): \xi \geqq 0\}$ is a semi-group of linear maps. Now

$$
\|W(\xi) x\|=\sup _{t \geqq 0}\left|x\left(q_{\xi}(t)\right)\right|=\sup _{t \in E_{\xi}}|x(t)|,
$$

where $E_{\xi}=q_{\xi}([0, \infty])$ is the range of $q_{\xi}$, which is contained in $[0, \infty]$. 
Thus the above norm is $\leqq \sup _{t \geq 0}|x(t)|=|| x \|$. Hence $\|W(\xi)\| \leqq 1$, and by choosing the element $x_{0} \in C[0, \infty]$ defined by $x_{0}(t)=1$, we see that $\left\|W(\xi) x_{0}\right\|=1$. Thus $W(\xi)$ is of norm 1 for each $\xi \geqq 0$. From this we conclude that the type of the semi-group $\mathfrak{B}$ is $0=\inf _{\xi>0} \xi^{-1} \log \|W(\xi)\|$, $([2], 306)$. Further, as the iterates of $q_{1}$ are continuous, we see that the function $\xi \rightarrow W(\xi)$ is continuous in the strong topology of operators on $C[0, \infty]$. The example $a(t)=1$, for which $q_{1}(t)=t+1$, shows that, in general, $\xi \rightarrow W(\xi)$ is not continuous in the uniform topology of operators. All of this goes to show that $\mathfrak{W}$ is a strongly continuous semi-group of operators of class (A) on $C[0, \infty],([2], 321)$.

Now let $D$ be the set of all functions $x \in C[0, \infty]$ for which the limit

$$
T x=\lim _{\xi \rightarrow 0+}(W(\xi) x-x) / \xi
$$

exists in the norm topology of $C[0, \infty]$. Equation (3) defines a (closed) linear transformation $T: D \rightarrow C[0, \infty]$, which is the infinitesimal generator of the semi-group $\mathfrak{W}$. Because of (2) it is seen that

$$
D=\left\{x \in C[0, \infty]: a x^{\prime} \text { exists as an element of } C[0, \infty]\right\},
$$

and so, for $x \in D$,

$$
(T x)(t)=a(t) x^{\prime}(t)
$$$$
t \geqq 0 .
$$

Because $\mathfrak{W}$ is of type 0 , the spectrum of $T$ is contained in the half-plane $\{z: \operatorname{Re}(z) \leqq 0\}$, and therefore, as a result of (4) and Theorem 11.5 .1 of [2], the solution of the first order differential equation

$$
-a x^{\prime}+\lambda x=f
$$

which belongs to $D$, for some given $f \in C[0, \infty]$, may be represented as a Bochner integral. But, by a similar argument to that used in [2] page 532, this may be interpreted as the following Lebesgue integral.

$$
x(t)=\int_{0}^{\infty} e^{-\lambda \xi} f\left(q_{\xi}(t)\right) d \xi
$$

for $t \geqq 0$ and for all $\lambda$ with positive real part. This representation of solutions of differential equations thus extends the theory of [2], page 532, which corresponds to the particular case $a(t)=1$.

A necessary condition for $q_{1}$ to generate a family of fractional iterates is that $q_{1}$ have a fixed point $\alpha \in[0, \infty]$. Thus $q_{1}(\alpha)=\alpha$, where possibly $\alpha=\infty$. As $q_{1}$ has $\alpha$ as a fixed point, so does each iterate $q_{\xi}, \xi \geqq 0$. We now see that (6) represents that unique solution of equation (5) which belongs to $D$ and which satisfies the boundary condition

$$
x(\alpha)=\int_{0}^{\infty} e^{-\lambda \xi} f\left(q_{\xi}(\alpha)\right) d \xi=f(\alpha) \int_{0}^{\infty} e^{-\lambda \xi} d \xi=f(\alpha) / \lambda .
$$

We summarize this result as 
THEOREM 1. Suppose that $a$ is positive and continuous on $[0, \infty]$, then that solution $x \in D$ of (5) which satisfies $x(\alpha)=f(\alpha) / \lambda$, is given by (6), at least when $\operatorname{Re}(\lambda)>0$.

At the expense of introducing 'almost everywhere' language, we can derive a solution similar to $(6)$ when $T$ acts on the domain $\left\{x \in L^{p}[0, \infty]\right.$ : $a x$ is absolutely continuous $\}$ in the Lebesgue spaces $L^{p}[0, \infty]$, for $p \geqq 1$.

We will illustrate the above representation by exhibiting a simple example. Choose $a(t)=e^{-t}$; we then find that $A(t)=e^{t}$, and, from equation (1), that $q_{\xi}(t)=\log \left(\xi+e^{t}\right)$. Thus that solution of

$$
-e^{-t} x^{\prime}(t)+\lambda x(t)=f(t)
$$

which has a continuous derivative, and which satisfies $x(\infty)=f(\infty) / \lambda$, is given by

$$
x(t)=\int_{0}^{\infty} e^{-\lambda \xi} f\left(\log \left(\xi+e^{t}\right)\right) d \xi,
$$

for $\operatorname{Re}(\lambda)>0$, and for each $f \in C[0, \infty]$.

Consider again the linear transformation $T: D \rightarrow C[0, \infty]$ as defined by (4). It is readily seen that $T^{2}$ is closed on its domain, and, ([1], 639), that it is the infinitesimal generator of a strongly continuous semi-group of operators $\{\Gamma(\xi): \xi \geqq 0\}$ over $C[0, \infty]$, of type 0 . In fact, this semi-group is given explicitly by, ([1], 638),

$$
(\Gamma(\xi) x)(t)=\frac{1}{\sqrt{\pi \xi}} \int_{0}^{\infty} e^{-8^{2} / 4 \xi} x\left(q_{s}(t)\right) d s .
$$

From this representation, we find that a solution $x$ of

$$
-a\left(a x^{\prime}\right)^{\prime}+\lambda x=f
$$

which belongs to $D\left(T^{2}\right)=\left\{x \in C[0, \infty]: a\left(a x^{\prime}\right)^{\prime}\right.$ exists and belongs to $C[0, \infty]\}$, is given by

$$
x(t)=\frac{1}{\sqrt{ } \pi} \int_{0}^{\infty} \xi^{-\frac{1}{2}} e^{-\lambda \xi} \int_{0}^{\infty} e^{-s^{2} / 4 \xi} f\left(q_{s}(t)\right) d s d \xi,
$$

for $\operatorname{Re}(\lambda)>0$.

Again we note that if $\alpha$ is a fixed point of the iterates $\left\{q_{\xi}\right\}$, then (8) satisfies $x(\alpha)=f(\alpha) / \lambda$, and, after a further laborious computation involving the use of Abel's equation (1), we find that if $f$ is differentiable at $\alpha$, then $x^{\prime}(\alpha)=f^{\prime}(\alpha) / \lambda$. We have therefore proven the

THEOREM 2. Let a be as in Theorem 1, and suppose that $f$ is differentiable. Then the solution $x$ of (7) which belongs to $D\left(T^{2}\right)$, and which satisfies $x(\alpha)=f(\alpha) / \lambda, x^{\prime}(\alpha)=f^{\prime}(\alpha) / \lambda$, is given by $(8)$ when $\operatorname{Re}(\lambda)>0$. 
In conclusion, we mention that the above method yields representations of solutions of certain initial-value problems for partial differential equations. We refer to [1], page 641 , for the relevant details.

I would like to thank Professor G. Szekeres for his many enlightening remarks on this paper.

\section{References}

[1] Dunford, N. and Schwartz, J. T., 'Linear operators'. Vol. I (Interscience, 1964, Second edition).

[2] Hille, E. and Phillips, R. S., 'Functional analysis and semigroups' (Amer. Math. Soc. Coll. XXXI, 1958).

[3] Szekeres G. 'Regular iteration of real and complex functions.' Acta Math. 100 (1958), $203-258$.

University of New South Wales

Kensington, N.S.W.

Australia 